Research Article

\title{
On the Change of Distance Energy of Complete Bipartite Graph due to Edge Deletion
}

\author{
Shaowei Sun (iD) and Ziyan Wan \\ School of Science, Zhejiang University of Science and Technology, Hangzhou, Zhejiang 310023, China \\ Correspondence should be addressed to Shaowei Sun; sunshaowei2009@126.com
}

Received 2 November 2020; Revised 20 December 2020; Accepted 24 December 2020; Published 9 January 2021

Academic Editor: Shaofang Hong

Copyright (C) 2021 Shaowei Sun and Ziyan Wan. This is an open access article distributed under the Creative Commons Attribution License, which permits unrestricted use, distribution, and reproduction in any medium, provided the original work is properly cited.

The distance energy of a graph is defined as the sum of absolute values of distance eigenvalues of the graph. The distance energy of a graph plays an important role in many fields. By constructing a new polynomial, we transform a problem on the sum of the absolute values of the roots of a quadratic polynomial into a problem on the largest root of a cubic polynomial. Hence, we give a new and shorter proof on the change of distance energy of a complete bipartite graph due to edge deletion, which was given by Varghese et al.

\section{Introduction}

Let $G=(V(G), E(G))$ be a simple connected graph, whose vertex set is $V(G)=\left\{v_{1}, v_{2}, \ldots, v_{n}\right\}$ and edge set is $E(G)$. For any pair of vertices $\left(v_{i}, v_{j}\right)$, the distance $d\left(v_{i}, v_{j}\right)$ is defined as the length of the shortest path between $v_{i}$ and $v_{j}$. In particular, $d\left(v_{i}, v_{i}\right)=0$ for any $v_{i} \in V(G)$. The distance matrix of $G$, denoted by $D(G)$, is the $n \times n$ matrix with $(i, j)$-entry being $d\left(v_{i}, v_{j}\right)$. The distance energy $E_{D}(G)$ of $G$ is defined by the sum of absolute values of distance eigenvalues of $G$. The distance energy of $G$ has been studied for several years in the literature, see [1-5] and the references therein.

It is not easy to compute the distance energy of general graphs. Thus, we are interested in finding the distance energy of some special graphs. For example, it has been proved in [6-8] that

$$
E_{D}\left(K_{n_{1}, n_{2}, \ldots, n_{k}}\right)=2\left(\sum_{i=1}^{k} n_{i}-k\right)
$$

where $K_{n_{1}, n_{2}, \ldots, n_{k}}$ is a complete $k$-partite graph with the size of each partition being at least 2 . The distance energy of some graphs with diameter 2 is also determined in [9]. Another interesting problem is to study how the distance energy of a graph changes by deleting an edge. Zhou and Ilić [10] showed that the deletion of any edge increases the distance energy of a connected graph with unique positive distance eigenvalue whenever the resulting graph is still connected. Varghese et al. [11] proved that $E_{D}\left(K_{p, q}\right)<E_{D}\left(K_{p, q}-e\right)$ for any edge $e$ of $E\left(K_{p, q}\right)$, where $K_{p, q}$ is a complete bipartite graph with $p, q \geq 2$. Recently, Tian et al. [12] showed that the deletion of any edge increases the distance energy of some special complete multipartite graphs.

In this paper, we will give a new and shorter proof of the main result in [11] by transforming a problem on the sum of the absolute values of the roots of a quadratic polynomial into a problem on the largest root of a cubic polynomial.

\section{Main Result}

In this section, we will give a new and shorter proof on $p, q \geq 2, E_{D}\left(K_{p, q}\right)<E_{D}\left(K_{p, q}-e\right)$, which has been proved in [11]. Before giving our main proof, we need a result on distance eigenvalues of a graph.

Lemma 1 (see [13]). Let $G$ be a graph of order n. If there exist $k$ vertices having the same neighborhood, then $G$ has distance eigenvalue -2 with multiplicity at least $k-1$. 
Next, we introduce a concept about the equitable quotient matrix. Let $M$ be a symmetric matrix of order $n$ whose block form is as follows:

$$
M=\left[\begin{array}{ccc}
M_{11} & \cdots & M_{1 t} \\
\vdots & \ddots & \vdots \\
M_{t 1} & \cdots & M_{t t}
\end{array}\right],
$$

where the blocks $M_{i j}$ are $n_{i} \times n_{j}$ matrices for any $1 \leq i, j \leq t$ and $n=n_{1}+\cdots+n_{t}$. Let $b_{i j}$ denote the sum of all entries in $M_{i j}$ divided by the number of rows. Then, $B(M)=\left(b_{i j}\right)$ is called the quotient matrix of $M$. If $M_{i j}$ has a constant row sum for each pair $(i, j)$, then $B$ is called the equitable quotient matrix of $M$. Haemers [14] obtained the relation between the spectra of $M$ and $B(M)$ as follows.

Lemma 2 (see [14]). Let $B$ be the equitable quotient matrix of $M$ as defined above. Then, any eigenvalue of $B$ is a eigenvalue of $M$.

Now, we are ready to give the main result of this paper.

Theorem 1. For integers $p, q \geq 2$ and $n=p+q$,

$$
E_{D}\left(K_{p, q}\right)<E_{D}\left(K_{p, q}-e\right)
$$

where $e$ is any edge of $K_{p, q}$.

Proof. If $n=4$ or 5, one can easily confirm that $E_{D}\left(K_{p, q}\right)<E_{D}\left(K_{p, q}-e\right)$ holds; otherwise, $n \geq 6$. From the structure of the graph $K_{p, q}-e$, by Lemma 1 , we found that the distance eigenvalues of $K_{p, q}-e$ are -2 with multiplicity $n-4$. Note that, after relabeling the matrix $D\left(K_{p, q}-e\right)$, we rewrite $D\left(K_{p, q}-e\right)$ as a $4 \times 4$ block matrix in the following form:

$$
M=\left[\begin{array}{cccc}
0 & 3 & 2 J_{1, p-1} & J_{1, q-1} \\
3 & 0 & J_{1, p-1} & 2 J_{1, q-1} \\
2 J_{p-1,1} & J_{p-1,1} & 2 J_{p-1, p-1}-2 I_{p-1} & J_{p-1, q-1} \\
J_{q-1,1} & 2 J_{q-1,1} & J_{q-1, p-1} & 2 J_{q-1, q-1}-2 I_{q-1}
\end{array}\right],
$$

where $J_{i \times j}$ be a $i \times j$ matrix with all entries being 1 . It is clear that each block of $M$ has a constant row sum. Thus, the equitable quotient matrix of $M$ is

$$
B=\left[\begin{array}{cccc}
0 & 3 & 2(p-2) & q-1 \\
3 & 0 & p-1 & 2(q-1) \\
2 & 1 & 2(p-2) & q-1 \\
1 & 2 & p-1 & 2(q-2)
\end{array}\right]
$$

After simple calculation, we obtain the characteristic polynomial of $B$ as

$$
\begin{aligned}
P(x)= & x^{4}-2(n-4) x^{3}+(3 p q-12 n+16) x^{2} \\
& +4(3 p q-4 n-4) x+12(n-5) .
\end{aligned}
$$

Since $P(-2)=-36 p q+108 p+108 q-140 \neq 0$ for any integer $p$ and $q, P(x)=0$ has four roots which are different from -2 . Combining these results with Lemma 2, we conclude that $D\left(K_{p, q}-e\right)$ has eigenvalue -2 with multiplicity $n-4$ and the remaining four eigenvalues (say, $\lambda_{1} \geq \lambda_{2} \geq \lambda_{3} \geq \lambda_{4}$ ) are the roots of $P(x)=0$. By Descartes' rule of signs with the fact that $\sum_{i=1}^{4} \lambda_{i}=2 n-8$, one can easily check that $\lambda_{1} \geq \lambda_{2}>0>\lambda_{3} \geq \lambda_{4}$. Now, let $a_{0},-a_{1}, a_{2}, a_{3}, a_{4}$ be the coefficients of $P(x)$. Note that $a_{0}=1$ and $a_{4}=12(n-5)$. Then, $P(x)$ can be rewritten as

$$
x^{4}-a_{1} x^{3}+a_{2} x^{2}+a_{3} x+a_{4}=\left(x^{2}-a x+b\right)\left(x^{2}+c x+d\right) \text {, }
$$

where $a_{1}=a-c, \quad a_{2}=b+d-a c, \quad a_{3}=b c-a d, \quad$ and $a_{4}=b d$.

Claim 1. $\left(\lambda_{1}+\lambda_{2}-\lambda_{3}-\lambda_{4}\right)^{2}-a_{1}^{2}>32 n-64$.

Proof of Claim. 1. From the given conditions, we have

$$
\begin{aligned}
(a+c)^{2}(b-d)^{2} & =(a+c)^{2}\left((b+d)^{2}-4 b d\right) \\
& =(a+c)^{2}\left(\left(a_{2}+a c\right)^{2}-4 a_{4}\right) \\
& =(a+c)^{2}\left(\left(a_{2}+\frac{(a+c)^{2}-a_{1}^{2}}{4}\right)^{2}-4 a_{4}\right) .
\end{aligned}
$$

On the other hand, we have

$$
(a-c)(b+d)=a_{1}\left(a_{2}+a c\right)=a_{1}\left(a_{2}+\frac{(a+c)^{2}-a_{1}^{2}}{4}\right) \text {. }
$$

As $2 a_{3}=2(b c-a d)$, we obtain

$$
2 a_{3}+(a-c)(b+d)=(a+c)(b-d) .
$$

Combining (8)-(10), we can obtain that $(a+c)^{2}-a_{1}^{2}$ is the root of $f(y)=0$, where

$$
\begin{aligned}
f(y)= & \frac{1}{16} y^{3}+\frac{a_{2}}{2} y^{2}+\left(a_{2}^{2}-4 a_{4}-a_{1} a_{3}\right) y \\
& -4 a_{1}^{2} a_{4}-4 a_{1} a_{2} a_{3}-4 a_{3}^{2} .
\end{aligned}
$$

From (7), it follows that $(a+c)^{2}$ has three possible values:

$$
\begin{aligned}
& \left(\lambda_{1}+\lambda_{2}-\lambda_{3}-\lambda_{4}\right)^{2}, \\
& \left(\lambda_{1}+\lambda_{3}-\lambda_{2}-\lambda_{4}\right)^{2}, \\
& \left(\lambda_{1}+\lambda_{4}-\lambda_{2}-\lambda_{3}\right)^{2} .
\end{aligned}
$$

Since $\lambda_{1} \geq \lambda_{2}>0>\lambda_{3} \geq \lambda_{4}$, then $\left(\lambda_{1}+\lambda_{2}-\lambda_{3}-\lambda_{4}\right)^{2}-a_{1}^{2}$ is the largest root of $f(y)=0$. Using the fact that $a_{3}=$ $4\left(a_{2}+8 n-20\right)$ and $a_{4}=12(n-5)$, we have 


$$
f(y)=\left(y-16 a_{1}-64\right) a_{2}^{2}+\left(\frac{y^{2}}{2}-4 a_{1} y-\left(64 a_{1}+512\right)(2 n-5)\right) a_{2}+g\left(n, y, a_{1}\right)
$$

where

$$
\begin{aligned}
g\left(n, y, a_{1}\right)= & \frac{y^{3}}{16}-16\left((2 n-5) a_{1}+3 n-15\right) y \\
& -64(8 n-20)^{2}-48(n-5) a_{1}^{2}
\end{aligned}
$$

Again using the fact that $a_{1}=2 n-8$, we obtain

$$
\begin{aligned}
f(32 n-64)= & 128 n a_{2}+g(n, 32 n-64,2 n-8) \\
= & 128 n a_{2}-192 n^{3}+1984 n^{2} \\
& -2048 n-1024 .
\end{aligned}
$$

As $p+q=n$, it follows that $a_{2} \leq\left(3 n^{2} / 4\right)-12 n+16$. Combining the above results, we have

$$
\begin{aligned}
f(32 n-64) \leq & 128 n\left(\frac{3 n^{2}}{4}-12 n+16\right)-192 n^{3} \\
& +1984 n^{2}-2048 n-1024 \\
= & -32(n-4)(n-2)(3 n+4)<0 .
\end{aligned}
$$

This follows directly that $\left(\lambda_{1}+\lambda_{2}-\lambda_{3}-\lambda_{4}\right)^{2}-a_{1}^{2}>$ $32 n-64$. This completes the proof of Claim 1 .

By Claim 1 with $a_{1}=2 n-8$, we obtain

$$
\lambda_{1}+\lambda_{2}-\lambda_{3}-\lambda_{4}>\sqrt{4(n-4)^{2}+32 n-64}=2 n \text {. }
$$

Hence, we have

$$
\begin{aligned}
E_{D}\left(K_{p, q}-e\right)= & 2(n-4)+\lambda_{1}+\lambda_{2} \\
& -\lambda_{3}-\lambda_{4}>4(n-2)=E_{D}\left(K_{p, q}\right) .
\end{aligned}
$$

This completes the proof of this theorem.

Remark 1. It is worth mentioning that the method we used in the above proof would be a tool to compare the sum of the absolute values of the roots of a quadratic polynomial with a certain value.

\section{Data Availability}

No data were used to support this study.

\section{Conflicts of Interest}

The authors declare that they have no conflicts of interest.

\section{Acknowledgments}

The first author was supported by the National Natural Science Foundation of China (Grant nos. 11901525 and 11801512) and Zhejiang Provincial Natural Science Foundation of China (LY20A010005).

\section{References}

[1] M. Aouchiche and P. Hansen, "Distance spectra of graphs: a survey," Linear Algebra and Its Applications, vol. 458, pp. 301-386, 2014.

[2] A. Ilić, "Distance spectra and distance energy of integral circulant graphs," Linear Algebra and Its Application, vol. 433, pp. 1005-1014, 2010.

[3] G. Indulal, "Distance spectrum of graph compositions," Ars Math. Contemp.vol. 2, pp. 93-100, 2009.

[4] H. S. Ramane, I. Gutman, and D. S. Revankar, "Distance equienergetic graphs," MATCH Communications in Mathematical and in Computer Chemistry, vol. 60, pp. 473-484, 2008.

[5] D. Stevanović and G. Indulal, "The distance spectrum and energy of the composition of regular graphs," Applied Mathematics Letters, vol. 22, pp. 1136-1140, 2009.

[6] W. So, "A shorter proof of the distance energy of complete multipartite graphs," Special Matrices, vol. 5, no. 1, pp. 61-63, 2017.

[7] D. Stevanović, M. Milošević, P. Híc, and M. Pokorný, "Proof of a conjecture on distance energy of complete multipartite graphs," MATCH Communications in Mathematical and in Computer Chemistry, vol. 70, pp. 157-162, 2013.

[8] X. Zhang, "The inertia and energy of distance matrices of complete graphs $k$-partite graphs," Linear Algebra and Its Applications, vol. 450, pp. 108-120, 2014.

[9] G. Indulal, I. Gutman, and A. Vijayakumar, "On distance energy of graphs," MATCH Communications in Mathematical and in Computer, vol. 60, no. 2, pp. 461-472, 2008.

[10] B. Zhou and A. Ilić, "On distance spectral radius and distance energy of graphs," MATCH Communications in Mathematical and in Computer Chemistry, vol. 64, pp. 261-280, 2010.

[11] A. Varghese, W. So, and A. Vijayakumar, "Distance energy change of complete bipartite graph due to edge deletion," Linear Algebra and Its Applications, vol. 553, pp. 211-222, 2018.

[12] G.-X. Tian, Y. Li, and S.-Y. Cui, "The change of distance energy of some special complete multipartite graphs due to edge deletion," Linear Algebra and Its Applications, vol. 584, pp. 438-457, 2020.

[13] P. Krrivka and N. Trinajstić, "On the distance polynomial of a graph," Aplikace Matematiky, vol. 28, pp. 357-363, 1983.

[14] W. H. Haemers, "Interlacing eigenvalues and graphs," Linear Algebra and Its Applications, vol. 226-228, pp. 593-616, 1995. 